\title{
Mitteilungen der DGMKG
}

MKG-Chirurg 2021 · 14:63-69

https://doi.org/10.1007/s12285-020-00286-y

(c) Springer Medizin Verlag GmbH, ein Teil von Springer Nature 2021

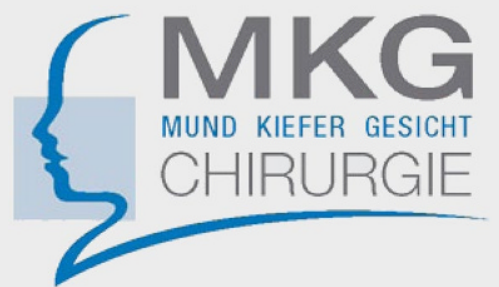

\author{
Redaktion \\ Dr. Jörg-Ulf Wiegner, Saalfeld \\ (v.i.S.d.P.)
}

Korrespondenzadresse

Deutsche Gesellschaft für Mund-, Kiefer- und Gesichtschirurgie e.V. Geschäftsstelle

Geschäftsführerin Kerstin Kothe

Schoppastraße 4, 65719 Hofheim

Tel. 06192-20 63 03, Fax 06192-20 6304

E-Mail: info@dgmkg.de

Internet: www.dgmkg.de

\section{Inhalt}

63 Deutscher Chirurgenkongress 2021 und Jahreskongress der DGMKG 2021

64 Bericht des Präsidenten der Deutschen Gesellschaft für Mund-, Kiefer- und Gesichtschirurgie für den Zeitraum 2019/2020

65 Bericht des Vizepräsidenten der Deutschen Gesellschaft für Mund-, Kiefer- und Gesichtschirurgie für den Zeitraum 2019/2020

66 Die wirtschaftliche Situation wissenschaftlicher Fachgesellschaften und Berufsverbände in Zeiten von COVID-19

67 Bericht aus dem Studienzentrum

68 Die Aktivitäten des Referats „Ästhetische Gesichtschirurgie" 69 Veranstaltungen

\section{Deutscher Chirurgenkongress 2021 und Jahreskongress der DGMKG 2021}

Die Planungen für den Deutschen Chirurgenkongress (DCK) 2021 und die Jahrestagung der Deutschen Gesellschaft für Mund-, Kiefer- und Gesichtschirurgie (DGMKG) 2021 werden weiterhin sehr stark von der COVID-19-Pandemie beeinflusst. Die in den letzten Wochen in Europa und auch in Deutschland stark steigenden Zahlen von Infizierten, verbunden mit Reisebeschränkungen und Beschränkungen im öffentlichen Leben, haben dazu geführt, dass verschiedene Mitgliedsgesellschaften der Deutschen Gesellschaft für Chirurgie (DGCH) die Meinung vertreten haben, dass ein DCK 2021 als Präsenzkongress nicht durchführbar sein wird.

Aufgrund dessen wurde in einer eigens dazu einberufenen
Vorstandssitzung der DGCH unter Beteiligung aller Mitgliedsgesellschaften beschlossen, vom Konzept des Präsenzkongresses 2021 abzuweichen und den DCK 2021 als Hybridkongress durchzuführen. Geplant sind 1-1,5 Tage Präsenzveranstaltungen, darunter die Kongresseröffnung, Mitgliederversammlungen, Gremiensitzungen und der Festvortrag.

Die wissenschaftlichen Inhalte inklusive der Industrieausstellung werden dann an etwa 4 Tagen ausschließlich online mit verschiedenen parallel stattfindenden Sitzungen abgehalten werden. Dieses Konzept wurde von allen Mitgliedsgesellschaften befürwortet und befindet sich derzeit in Planung. Aufgrund der veränderten techni- schen Rahmenbedingungen für einen Onlinekongress wurde der Kongressort von der Messe München in das Kongresszentrum Mainz inklusive der Rheingoldhalle verlegt. Der ursprünglich für den Kongress vorgesehene Zeitpunkt, 12.-16. April, bleibt bestehen. Gemeinsam mit der Deutschen Gesellschaft für Allgemein- und Viszeralchirurgie (DGAV) und der Deutschen Gesellschaft für Kinderchirurgie (DGKCH) ist die DGMKG Mitveranstalter des DCK 2021.

Auch die Industrie wird mit eigens für das webbasierte Format angepassten Präsentationen am Kongress teilnehmen; möglich sind virtuelle Ausstellungsstände, aber auch themenbezogene Präsentationen.

Der anstehende Hybridkongress ist ein Zeichen, Herausforderung anzunehmen und dabei moderne Wege der Kommunikationstechnik zu nutzen. Durch dieses neue Kongressformat besteht die Chance, die wissenschaftliche Präsenz aller chirurgischen Fächer, aber insbesondere der DGMKG, auch unter schwierigen Bedingungen hochzuhalten.

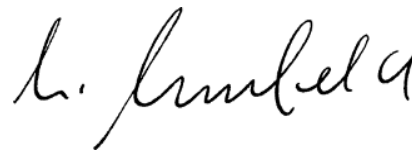

Univ.-Prof. Dr. Dr. Michael Ehrenfeld Kongresspräsident DCK, Präsident DGCH Klinik und Poliklinik für Mund-, Kiefer- und Gesichtschirurgie, Klinikum der Universität München

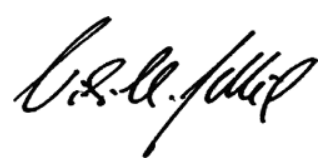

Univ.-Prof. Dr. Dr. Nils-Claudius Gellrich Kongresspräsident DGMKG Klinik und Poliklinik für Mund-, Kiefer- und Gesichtschirurgie, Medizinische Hochschule Hannover (MHH)

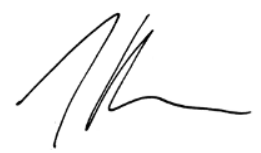

Univ.-Prof. Dr. Dr. Jürgen

Hoffmann

Präsident DGMKG

Klinik und Poliklinik für Mund-, Kiefer- und Gesichtschirurgie, Universitätsklinikum Heidelberg 


\section{Bericht des Präsidenten der Deutschen Gesellschaft für Mund-, Kiefer- und Gesichtschirurgie für den Zeitraum 2019/2020}

\begin{abstract}
Zum Zeitpunkt der Erstellung dieses Berichtes waren 1956 Kollegen Mitglied der DGMKG. Davon arbeiten 405 Fachärzte an Kliniken, 175 Kollegen als Weiterbildungsassistenten, 978 als niedergelassene MKG-Chirurgen und 97 als angestellte Kollegen bei MKG-Chirurgen; 251 sind Pensionäre und 26 Kollegen ohne Anstellung. Der Arbeitskreis Wissenschaft (AKWi) hat 364 Mitglieder. Damit verzeichnen wir mit plus $1,5 \%$ eine positive Mitgliederentwicklung im Berichtszeitraum.
\end{abstract}

\section{Muster- und Zusatzweiter- bildungsordnung}

Nachdem die Musterweiterbildungsordnung MKG-Chirurgie sowie die uns betreffende $\mathrm{Zu}$ satzweiterbildungsordnung Plastische- und Ästhetische Operationen auf dem Ärztetag 2018 in Erfurt verabschiedet wurden, stand die Umsetzung der Musterweiterbildungsordnung in die Weiterbildungsordnungen der Landesärztekammern im Mittelpunkt des Interesses. Es freut mich außerordentlich, Ihnen mitteilen zu können, dass wir über die 12-monatige Anerkennung oralchirurgischer Weiterbildungen vor ärztlicher Approbation, wie sie in der Musterweiterbildungsordnung geregelt ist, in den Kammerbereichen Baden/Württemberg, Nordrhein, Westfalen und Thüringen weitere 12 Monate Anerkennungsfähigkeit verankern konnten.

Voraussetzung für die Anerkennung ist, dass der Weiterbildungsermächtigte sowohl für die Oralchirurgie als auch die MKGChirurgie benannt ist. Zukünftig wird es leider in Deutschland kein einheitliches Bild bezüglich der Anerkennungsfähigkeit dieser zahnmedizinischen Weiterbildungsinhalte geben können.

Erfreulicherweise konnten einige für uns wesentliche Aspekte der auf dem Deutschen Ärztetag in Erfurt verabschiedeten Musterweiterbildungsordnung mit der Bundesärztekammer adaptiert werden. Darunter zählte u.a. die Neuformulierung zur Berechtigung der Ausübung der Chirurgie der Körperoberfläche.

Auf der Grundlage dieser neuen Formulierung in der Musterweiterbildungsordnung haben wir zunächst in einem Bundesland beginnend den Versuch unternommen, auf Ebene der kassenärztlichen Vereinigung die Fachgebietsbeschränkungen bezüglich dieses Tätigkeitsschwerpunktes einer neuen Bewertung zu unterziehen. Mit Unterstützung des Vorstandes ist es uns im Schulterschluss mit der HalsNasen-Ohren-Heilkunde (beide Fachgruppen verfügen über eine nahezu wortidentische Formulierung zur Körperoberflächenchirurgie in der Musterweiterbildungsordnung) gelungen, die Fachgebietsgrenzen in diesem Punkt aufzuheben.

\section{Fortbildungsstrukturen}

Derzeit bemüht sich der Vorstand mit dem wissenschaftlichen Leiter der Akademie, Herrn Prof. Ehrenfeld, dem Geschäftsführer der Europäischen Fortbildungsakademie für Medizin und Zahnmedizin (EFMZ), Herrn Prof. Wiltfang, dem wissenschaftlichen Leiter der EFMZ, Herrn Prof. Haßfeld, und dem Fortbildungsreferenten der DGMKG, Herrn Dr. Bons- mann, gemeinsam mit den Referatsleitern, Herrn Dr. Kettner und Herrn Dr. Ulrich, um eine Zusammenführung der Fortbildungsstrukturen der DGMKG. In der neuen Struktur werden wir die gesamten Fortbildungsaktivitäten der DGMKG bündeln und ein an den Bedürfnissen unserer Mitglieder orientiertes Konzept etablieren. Der neu gegründete Satzungsausschuss der DGMKG befasste sich in den letzten beiden Jahren ebenfalls intensiv mit den Erfordernissen innerhalb der Neustrukturierung der Fortbildung der DGMKG. Hier werden wir einen ersten Vorschlag anlässlich einer Onlinevorstandssitzung im Vorfeld der Mitgliederversammlung vorstellen und diskutieren.

Im Februar 2020 wurde nach einstimmiger Beschlussfassung im Vorstand das erste eigenständige Praxisführungsseminar in Frankfurt/Höchst nach 2015 durchgeführt. Die Inanspruchnahme lag deutlich über den Erwartungen. Hier wurden und werden neue Fortbildungsformate etabliert, die auf breite Resonanz bei den niederlassungswilligen und niedergelassenen Kollegen trafen.

\section{Versorgungsforschung/ Studienzentrum}

In dem Bemühen als DGMKG, trotz ihrer vergleichsweise geringen Mitgliederzahl auch nach außen auf Studienebene besser sichtbar zu werden, etablierte der Vorstand der DGMKG einen Lenkungsausschuss, um Studienaktivitäten sowohl zu Fragen der Versorgungsforschung als auch zu Fragen der klinischen Forschung zusammenzuführen und zu koordinieren. Unter der Leitung von Herrn Prof. Heiland konnten zwischenzeitlich mehrere multizentrische Studien konzipiert werden. Für mehrere Studien liegen auch die notwendigen Ethikvoten vor, sodass in Kürze mit der Patientenakquise begonnen werden kann. Im Namen des gesamten Lenkungsausschusses bitte ich unsere Mitglieder um zahlreiche Teilnahme.

\section{Hauptstadtrepräsentanz}

Im Berichtszeitraum konnte die Hauptstadtrepräsentanz personell untersetzt werden. Frau Dettmann steht zur Koordination von so wichtigen Aufgaben wie u.a. Europavertretung, Leitlinienarbeit und Fortbildungskoordination unseren Mitgliedern zur Verfügung.

\section{Jahreskongress}

Der wissenschaftliche Jahreskongress unserer Fachgesellschaft im Juni 2020 in Hamburg unter der Leitung unseres Kongresspräsidenten Herrn Prof. Wiltfang und dessen Team aus Kiel musste durch die COVID-19-Pandemie bedingt abgesagt werden - eine Entscheidung, die dem Vorstand außerordentlich schwergefallen ist. Es ist für mich von großer Bedeutung, auf der einen Seite den Organisatoren für ihren Einsatz herzlich zu danken, auf der anderen Seite aber auch unseren Mitgliedern für deren Verständnis.

Ein kleines Detail der Kongressplanung konnten wir mit Unterstützung des Springer Medizin Verlags dennoch realisieren. Die von Herrn Prof. Wiltfang angeregten Präsentationen zur Historie unserer Gesellschaft wurden professionell aufgezeichnet und stehen den interessierten Mitgliedern im geschlossenen Bereich unserer Homepage zur Verfügung.

Wie unseren Mitgliedern bekannt, sollte der Jahreskongress der DGMKG 2021 im Rahmen des DCK 2021 unter der Leitung von Herrn Prof. Gellrich und dessen Team aus Hannover in München stattfinden. Auch hier stehen wir in ständigem Kontakt zur DGCH unter der Präsidentschaft von Herrn Prof. Ehrenfeld. Der derzeitige Planungsstand geht von einer hybriden Organisationsstruktur des DCK 2021 mit Präsenzteil in Mainz aus, wie sie dem Eingangsbericht der Mitteilungsseiten entneh- 
men können. Die Präsentation des diesjährigen Wissenschaftspreisträgers unserer Gesellschaft erfolgt anlässlich dieses Kongresses.

\section{Europa}

Im Januar 2019 stand der Vorstand vor der großen Herausforderung, die Europarepräsentanz unserer Fachgruppe neu ordnen zu müssen. Mit Herrn Dr. Ulrich konnten wir einen erfahrenen Vertreter unseres Faches als Secretary der Section Oral and Maxillofacial Surgery (OMFS) in die European Union of Medical Specialists (UEMS) einbringen. Mit Herrn Dr. Merholz stellen wir derzeit den Präsidenten der European Association of Senior Hospital Physicians (AEMH). Die Wahrung unserer Interessen mit dem Erhalt der Doppelapprobation und der Verteidigung der Fachgebietsgrenzen besitzen auf europäischer Ebene eine große Bedeutung.

\section{Satzungsänderung}

Ein Hauptschwerpunkt in der laufenden Legislatur stellte die Entwicklung einer Satzungsänderung der DGMKG dar. Der Vorstand hatte sich dazu entschlossen, einen Satzungsausschuss zu gründen, in dem die inhaltlichen und juristischen Unschärfen unserer Satzung an die aktuellen Gegebenheiten nach 20 -jähriger erfolgreicher Arbeit auf Basis der bestehenden Satzung angepasst werden sollten. Im Rahmen der sich daraus ergebenden Diskussionen können wir derzeit noch kein finales Ergebnis präsentieren. Wir planen, einen breit konsentierten Vorschlag anlässlich der Mitgliederversammlung auf dem Jahreskongress 2022 in Dresden präsentieren zu können.

\section{Approbationsordnungen}

Informationen zu diesem für uns wichtigen Arbeitsschwerpunkt entnehmen Sie bitte dem Bericht des Vize-Präsidenten, Herrn Prof. Hoffmann.

\section{Liebe Kolleginnen, liebe Kollegen,}

gern wiederhole ich Ausführungen aus dem letztjährigen $\mathrm{Be}$ richt: Die Vorstandsarbeit war geprägt von gegenseitigem Vertrauen. Ich möchte mich an dieser Stelle explizit bei dem Vizepräsidenten unserer Gesellschaft, Herrn Prof. Hoffmann, für die sehr gute Zusammenarbeit bedanken. Auf dieser Basis konnten wir uns den vielfältigen Sachaufgaben zuwenden, die ein hohes $\mathrm{Ma}$ an Engagement eines jeden Vorstandsmitgliedes forderten. Allen Mitgliedern danke ich herzlich für das Vertrauen in den Vorstand und in meine Person. Ein großer Dank gilt allen Mitgliedern unserer Gesellschaft, die sich aktiv für die Interessen der DGMKG eingesetzt haben und weiter einsetzen werden. Ohne das vielfältige Engagement unserer ehrenamtlich tätigen Mitglieder wären die nachweisbaren Erfolge nicht zustande gekommen. In der Vorfreude auf die vor uns stehenden Aufgaben und mit der Versicherung einer konsequent lösungsorientierten Vorstandsarbeit verbleibe ich mit freundlichen kollegialen Grüßen.

\section{Dr. Jörg-Ulf Wiegner}

Präsident der DGMKG

2018-2020

Saalepraxis - Praxis für MundKiefer- und Gesichtschirurgie, Oralchirurgie, Saalfeld

\section{Bericht des Vizepräsidenten der Deutschen Gesellschaft für Mund-, Kiefer- und Gesichtschirurgie für den Zeitraum 2019/2020}

Sehr geehrte Kolleginnen und Kollegen,

liebe Mitglieder,

Sie entnehmen bereits dem Bericht des Präsidenten, dass der Vorstand im Berichtszeitraum in gemeinsamer Verantwortung sehr umfangreich für die Fachgesellschaft tätig war. Daher bitte ich, meinen Bericht als thematische Ergänzung zu verstehen.

Die Tätigkeit des Vorstands war durch die umfangreiche Vernetzung mit anderen Fachgesellschaften geprägt, die unser Fach bei vielen Gelegenheiten sichtbarer macht und uns die Unterstützung bei interdisziplinär getragenen Entscheidungen ermöglicht:

\section{- Deutsche Gesellschaft für}

Chirurgie (DGCH): Sowohl unser ehemaliger Präsident,

Herr Prof. Ehrenfeld, wie auch der Unterzeichner sind Mitglieder des Präsidiums der DGCH. Wir nehmen dort eine hohe Anerkennung der Leistungen unseres Fachs wahr. Am 01.07.2020 wurde Herr Prof. Ehrenfeld zum Präsidenten der DGCH ernannt, womit die MKG-Chirurgie weiteren Respekt im chirurgischen Fächerkanon erfährt.

Wir haben im Berichtszeitraum regelmäßig im Rahmen des Chirurgenkongresses vorzugsweise interdisziplinär orientierte Sitzungen veranstaltet, um den Austausch zwischen den beteiligten Fächern zu unterstützen. In diesem Zusammenhang hat auch die Einrichtung einer weiteren Geschäftsstelle im Langenbeck-Virchow-Haus, mithin auf dem Campus der Charité, eine signifikante Be- deutung. Diesbezüglich sowie hinsichtlich des geplanten gemeinsamen Kongresses sei auf den Bericht des Präsidenten verwiesen.

- Dachverband Osteologie (DVO): Der Vorstand hat in seiner Klausur 07/2019 entschieden, dem Dachverband Osteologie (https://dv-osteologie.org) beizutreten. Für die Vertretung der Interessen unserer Fachgesellschaft wurden Herr Prof. Dr. Dr. Otto (Halle) sowie Herr PD Dr. Dr. Ristow (Heidelberg) mandatiert.

Über die Vertretung im Dachverband wurden wir bereits an der Entwicklung der für unser Fachgebiet relevanten Leitlinien beteiligt. Ferner sind wir mit eigenen Sitzungen am interdisziplinär orientierten Osteologiekongress vertreten und bieten Webinare zu relevanten Themen an. Eine besondere Bedeutung hatte die Vertretung der DGMKG im DVO zudem im Rahmen des vom Gemeinsamen Bundesausschuss verantworteten $\mathrm{Di}$ sease Management Programm „Osteoporose“.

- Deutsche Krebsgesellschaft (DKG) und Interdisziplinäre Arbeitsgruppe KopfHals-Tumoren (IAG-KHT): Der Vorstand hat im Berichtszeitraum die Vertretung unseres Fachs in den Zertifizierungskommissionen für Kopf-, Hals- und Hauttumor-Zentren koordiniert. Wir haben uns zudem erfolgreich um eine Beteiligung in der Zertifizierungskommission für die Sarkomzentren bemüht. Damit 
gelingt uns eine konsequente Berücksichtigung unseres Fachs im Rahmen der onkologischen Behandlung. Mit der Vertretung im Vorstand der IAG-KHT findet zudem ein interdisziplinärer Austausch mit den an der Tumortherapie beteiligten Fächern statt. In Abstimmung mit der DKG war damit auch eine erneute relevante Vertretung der MKGChirurgie im Rahmen des Deutschen Krebskongresses 2020 möglich.

In Abstimmung mit dem Vorstand des Deutsch-Österreichisch-Schweizerischen Arbeitskreises für Tumoren des Kiefer-Gesichtsbereichs (DÖSAK) streben wir zudem unter Berücksichtigung des vom Bundesministerium für Gesundheit (BMG) erneut etablierten Nationalen Krebsplans die Einrichtung eines Krebsregisters für die in unserem Fach relevanten Entitäten an. Hierzu erfolgt seit geraumer Zeit eine enge Abstimmung mit der Arbeitsgemeinschaft Deutscher Tumorzentren.

Mit der Entscheidung des Bundesrats, die Approbationsordnung für Zahnärzte (AOZ) zumindest teilweise zu novellieren, hat sich im Wettbewerb der Lehrfächer in der Zahnheilkunde die Frage der zukünftigen Verteilung notwendiger Ressourcen ergeben. Unter Einbeziehung des Vorstands der Vereinigung für Hochschullehrer in der ZMK (VHZMK) sowie des Medizinischen Fakultätentags haben wir 10/2019 der Etablierung eines unter den Fächern nicht konsentierten Musterstundenplans widersprochen und eine zunächst ergebnisoffene Diskussion der Verteilung der Lehrdeputate eingefordert.

Zur Umsetzung der zunächst für den klinischen Teil der zahnärztlichen Ausbildung relevanten Anteile wurde bereits 11/2018 im Arbeitskreis Wissenschaft unserer Fachgesellschaft eine Arbeitsgemeinschaft Lehre etabliert, die zunächst von Herrn Prof. Sader
(Frankfurt) geleitet wurde. Seit 05/2019 trägt Herr Prof. Al-Nawas (Mainz) die Verantwortung für die Vertretung unserer Interessen im Bereich der human- und zahnmedizinschen Lehre.

Eine auf die Klinik beschränkte Umsetzung der AOZ war zunächst angedacht, um eine Anpassung an die Änderung der Studienstruktur im Rahmen der ärztlichen Ausbildung (zunächst „Masterplan 2020“) berücksichtigen zu können.

Wir sind nun in Abstimmung und mit Unterstützung der Vorstände der VHZMK, der Bundeszahnärztekammer sowie der Deutschen Gesellschaft für Zahn-, Mund- und Kieferheilkunde darum bemüht, einen weitestgehenden Abgleich der Lehrinhalte der ersten Semester (bislang „Vorklinik“) zu begründen. Sowohl die Arbeitsgemeinschaft der Wissenschaftlichen Medizinischen Fachgesellschaften, der Spitzenverband der Fachärzte in Deutschland wie auch die DGCH stärken uns bei diesem Vorhaben den Rücken.

Ein Abgleich der Studieninhalte zeigt, dass eine weitestgehende gemeinsame Lehre in den Fächern Human- und Zahnmedizin möglich sein kann. Mit dem wesentlichen Einfluss unseres Hauptstadtrepräsentanten, Herrn RA Milkereit, hatten wir die Möglichkeit, dieses Konzept im November 2020 dem Leiter des zuständigen Referats im BMG vorstellen zu dürfen.

Zuletzt will ich über die Bemühungen unseres Vorstands zur Verbesserung der Kommunikation mit den Mitgliedern sowie zur Optimierung der Außendarstellung unserer Fachgesellschaft sowie unseres Fachs berichten.

Wir diskutieren seit geraumer Zeit die Notwendigkeit einer Aktualisierung unseres Internetauftritts. Mit tratkräftiger Unterstützung durch das Junge Forum sowie der Mitarbeiterinnen unserer Geschäftsstellen in Hofheim (Frau Weihrich) und Berlin (Frau Dettmann) erfolgte eine Analyse des Bedarfs sowie ein Vergleich der Angebote verschiedener Dienstleister.
Wir müssen nun feststellen, dass eine Kontrolle der für dieses Projekt ggf. eskalierenden Kosten nur durch einen modularen Aufbau, d.h. durch ein schrittweises Vorgehen, möglich sein wird. Wir wollen hierbei zudem ein besonderes Augenmerk auf die Integration sozialer Medien sowie Imagekampagnen legen, um ein möglichst großes Zielpublikum zu erreichen.

Mit der Etablierung eines regelmäßig sowie anlassbezogen erscheinenden Newsletters ist es uns sicherlich gelungen, Sie zeitnah über wichtige Themen zu informieren.

Meine Ausführungen wie auch die Berichte der übrigen Vorstandsmitglieder beschreiben nur orientierend, mit welchen Aufgaben der Vorstand unserer Fachgesellschaft betraut ist. Eine besondere Herausforderung stellen hierbei die Informationsdichte und -frequenz sowie die Diversifikation der bearbeiteten Themen dar.

Wir können dies nur mit einer hervorragenden Unterstützung durch die Beiräte, Arbeitskreise und Referate sowie insbesondere der Geschäftsstellen bewältigen.

Hierfür möchte ich den Mitarbeiterinnen und Mitarbeitern sowie Kolleginnen und Kollegen meinen herzlichen Dank aussprechen.

Mit freundlichen kollegialen Grüßen,

\section{Univ.-Prof. Dr. Dr. Jürgen Hoffmann} Vizepräsident DGMKG 2018-2020

Klinik und Poliklinik für Mund-, Kiefer- und Gesichtschirurgie, Universitätsklinikum Heidelberg

\section{Die wirtschaftliche Situation wissenschaftlicher Fachgesellschaften und Berufsverbände in Zeiten von COVID-19}

Die weltweite COVID-19-Pandemie sowie die für nötig erachteten Maßnahmen wie Lockdown, Quarantäne, Einschränkung der Reisetätigkeit etc. haben uns alle sowohl im privaten als auch im beruflichen Umfeld massiv getroffen. Selbstverständlich gilt dies auch für wissenschaftliche Fachgesellschaften und Berufsverbände, die ebenso wie wir alle auf eine derartige Dramatik nicht vorbereitet waren. Die sog. erste Welle hat Veranstaltungen und Kongresstermine zunächst zu Zitterpartien werden lassen, um es dann dennoch erforderlich zu machen, dass diese, da jedweder persönliche Kontakt einzustellen war, abgesagt werden mussten.
In den Verträgen mit Kongressveranstaltungsagenturen, Hotels und Cateringunternehmen war der Pandemiefall verständlicherweise nicht oder nur unpräzise definiert. Gleiches gilt für staatliche Ausgleichshilfen im Fall der verordneten Versammlungsverbote.

Der Vorstand unserer DGMKG hat sich bzgl. des für Juni 2020 in Hamburg geplanten Jahreskongresses, auch um Planungssicherheit für unsere Kollegen und Industriepartner zu gewährleisten, entschlossen, das wirtschaftliche Risiko einer frühzeitigen Absage zu übernehmen und den Großteil der Verlustsumme aus der unvermeidbaren Kongressabsage ggf. selbst zu schultern, was aufgrund 
einer vorausschauend vorsichtigen Finanzpolitik der letzten Jahre möglich gewesen wäre. Durch geschicktes Verhandeln der betreuenden Kongressagentur sowie eine solidarische Zusammenarbeit aller Beteiligten konnten die Defizite bilanztechnisch weitestgehend gegengerechnet werden.

Im weiteren Verlauf hat sich als hilfreich erwiesen, dass sich unsere DGMKG im Gegensatz $\mathrm{zu}$ anderen sehr großen Fachgesellschaften in erster Linie durch Mitgliedsbeiträge finanziert und damit weniger abhängig ist von Einnahmen durch Kongress- und Fortbildungsveranstaltungen sowie Industriesponsoring.

Dennoch machte es der fast vollständige Ausfall von Einnahmen durch Fortbildungsveranstaltungen, Kongresse etc. für das Jahr 2020 erforderlich, die Kalkulation für 2021 deutlich reduzierter anzugehen.

Während der Sommermonate keimte die zarte Hoffnung auf eine reduzierte Veranstaltungstätigkeit in der 2. Jahreshälfte unter Berücksichtigung der jeweiligen Hygienerichtlinien, was mit der sog. 2. Welle und dem dadurch stattfindenden Lockdown konterkariert wurde. Mittlerweile bestehende Erfahrungen und infrastrukturelle Konzepte für webbasierte Veranstaltungen scheinen aber einen, wenn auch deutlich veränderten, Kongress- und Veranstaltungsbetrieb wieder möglich zu machen.

Keinerlei Erfahrungen und Erkenntnisse bestehen augenblicklich noch darüber, inwieweit diese veränderte Art der wissenschaftlichen und standespolitischen Kommunikation von unseren Mitgliedern akzeptiert wird, wo- bei sicher davon auszugehen ist, dass sich in Bezug auf pandemiebedingte Einschränkungen von Großveranstaltungen bis weit in das Jahr 2021 nichts Wesentliches ändern wird. Erstmalig ist deshalb auch der Haushaltsentwurf der DGMKG für das Jahr 2021 in zwei unterschiedlichen Varianten präsentiert worden, was ebenso wie die Durchführung einer webbasierten Mitgliederversammlung mit Vorstandswahlen eine absolute Novität darstellt.

Parallel zum Rückgang der Einnahmen sind aber auch Ausgaben reduziert angefallen, was auf das pandemieverursachte Einstellen jedweder Reisetätigkeit, ausschließlich webbasierte Sitzungstätigkeit, effektiv digitalisierte Kommunikationswege etc. zurückzuführen ist, sodass die Abschlussbilanz für das Katastrophenjahr 2020 weitestgehend ausgeglichen ausfallen dürfte.

Für 2021 ist existenziell wichtig, dass unsere Mitglieder der DGMKG weiter verbunden bleiben, die veränderte, nun meist digitale Qualität von Serviceleistungen, Fortbildungsangeboten und Kommunikationsplattformen unterstützend akzeptieren, wie z.B. anlässlich des online durchgeführten Curriculums Implantologie mit mehr als 100 Teilnehmern im November 2020 bereits geschehen, damit im Rückblick die Jahre 2020/2021 als solidarisch bewältigte Krise und genutzte Chance einer Neuorganisation in Erinnerung bleiben können.

\section{Florian Kubitzek}

Schatzmeister der DGMKG

Praxis für Mund-, Kiefer- und Gesichtschirurgie, München

\section{Bericht aus dem Studienzentrum}

Am 22.11.2019 tagte der Lenkungsausschuss in Berlin und diskutierte intensiv die Studien- beide Studien durch eine an der Universitätsklinik Frankfurt vorhandene Infrastruktur als Studienzentrale betreuen zu lassen und für beide Studien zunächst Ethikvoten in Frankfurt einzuholen. Auch wurde intensiv beraten, dass eine derartige Betreuung seitens der DGMKG finanziell unterstützt werden müsste.

Die nächste Sitzung des Lenkungsausschusses fand dann als Online-Meeting am 20.05.2020 statt. Das Studienprotokoll der Hautkrebsstudie konnte mittlerweile finalisiert werden. Die Teilnahme an dieser Studie, für die seinerzeit 85 Kolleginnen und Kollegen ihr Interesse an einer Teilnahme geäußert hatten, soll bundesweit für Niedergelassene als auch Kliniken möglich sein. In Vorbereitung, um das Ethikvotum aus Frankfurt dann entsprechend bei anderen Landesärztekammern (LÄK) einreichen zu können, verlangte die Frankfurter Ethikkommission die Benennung von 5 konkreten Kooperationspartnern. Da dasselbe für die Socket-Preservation-Studie verlangt wurde, wurde beschlossen, für beide Studien dieselben Kooperationspartner zu benennen. Für die Dokumentation der Hautkrebsstudie wurde das Online-Tool secuTrial getestet und für tauglich befunden, die Dokumentation der Socket-Preservation-Studie wird in impDAT erfolgen. Als Anschubfinanzierung für die Initiierung und Betreuung während der ersten 12 Monate werden Kosten von 60.000 Euro konkret dargestellt.

Von den auf dem Jahreskongress in Frankfurt vorgestellten Studien wurden seinerzeit eine Studie zur Vorhersage der malignen Transformation mithilfe eines neuartigen ImmunoScores aus Erlangen (Ansprechpartner: Dr. Dr. Weber, MKG, Uni Erlangen) und eine Beobachtungsstudie nach chirurgischer Therapie von Keratozysten aus Lübeck (Ansprechpartnerin: Dr. Cohrs, MKG, Uni Lübeck) als am interessantesten bewertet. Beide Studien wurden diskutiert und jeweils als "Studie im Interesse der DGMKG“ eingeordnet, wobei beide Studien kein finanzielles Engagement der DGMKG erfordern und als Multicenterstudien prinzipiell allen Mitgliedern in Absprache mit den Studienzentralen zur Teilnahme verfügbar sind.

Die nächste Sitzung des Lenkungsausschusses fand dann wiederum als Online-Meeting am 29.09.2020 statt. Frau Dr. Cohrs aus Lübeck stellte als Studienleiterin detailliert die Keratozystenstudie vor. Bis zu diesem Zeitpunkt hatten bereits 4 Praxen und 14 Kliniken ihr Interesse an einer Teilnahme geäußert, das positive Ethikvotum aus Lübeck liegt bereits vor. Kritisch werden die Kosten der erforderlichen Referenzpathologie aus Basel, die bei jedem Fall einzuholen ist, diskutiert. Herr PD Dr. Dr. Ristow aus Heidelberg stellte die APOS-Studie vor, die prospektiv und verblindet randomisiert den Nutzen einer prolongierten Antibiotikaprophylaxe bei Dysgnathieoperationen untersuchen wird. Die Studie wird von der Deutschen Forschungsgemeinschaft gefördert und wurde als „Studie im Interesse der DGMKG“ eingeordnet. Kritisch für die Teilnahme von Interessenten wurden die Dauer der postoperativen stationären Medikamentengabe und die Notwendigkeit einer Apotheke mit Studienerfahrung gesehen.

Für die Hautkrebsstudie liegt mittlerweile ein positives Ethikvotum in Frankfurt vor, das nun über die Vorstände der Landesverbände zusammen mit Universitätskliniken bei den einzelnen LÄK eingereicht werden soll. Die Kosten für derartige Ethikvoten werden von der DGMKG übernommen. Als weiterer und neuer Punkt wurde im Lenkungsausschuss eine Initiative des Spitzenverbandes Fachärzte Deutschlands im Feld der Versorgungsforschung diskutiert und eine Handlungsnotwendigkeit für die DGMKG klar bejaht.

Liebe Kolleginnen und Kollegen, ich freue mich, dass der Lenkungsausschuss mit Leben gefüllt werden konnte und begonnen hat, und der Socket-Preservation-Studie. Prinzipiell wurde angestrebt, 
die wissenschaftliche Sichtbarkeit unserer Fachgesellschaft zu stärken. Besonders positiv empfinde ich, dass die beiden von der DGMKG auch finanziell zu unterstützenden Studien sowohl niedergelassene als auch in Kliniken tätige Kolleginnen und Kollegen zur Teilnahme einladen, insbesondere vor dem Hintergrund, dass beide Fragestellungen für

\section{Die Aktivitäten des Referats „Ästhetische Gesichtschirurgie"}

\begin{abstract}
Ästhetik und Funktion gehen Hand in Hand in der Mund-Kiefer- und Gesichts(MKG)-Chirurgie und sind in allen Kerndisziplinen eng miteinander verknüpft. Als neuer Leiter des Referats „Ästhetische Gesichtschirurgie" der DGMKG ist mir folglich die Bedeutung der Ästhetik in der MKG-Chirurgie ein besonderes Anliegen.
\end{abstract}

\section{Mein Weg in die Ästhetik durch die MKG-Chirurgie}

Die ästhetische Gesichtschirurgie begleitet mich schon seit frühen Kindertagen - sie wurde mir quasi in die Wiege gelegt. Schon mein Vater hatte sich mit Begeisterung der ästhetisch-plastischen Chirurgie gewidmet. Unsere Klinik und das Zuhause befanden sich in einem Gebäude und so wuchs ich praktisch mit der Arbeit meines Vaters sehr eng verbunden auf. So kam ich schon sehr früh mit diesem Fachgebiet in Kontakt und die Faszination ist bis heute geblieben. Ein Schlüsselerlebnis war die Liveoperation des angesehenen MKG-Chirurgen Prof. Dr. Dr. Horst Günther aus Hamburg in unserer Klinik, bei der dieser eine Kinnosteotomie demonstrierte. Ab diesem Moment war für mich klar, dass auch ich in die Ästhetik über den Weg der MKG-Chirurgie gehen unser Fachgebiet auch berufspolitisch relevante Fragen betreffen. ne zur Verfügung.

\section{Mit freundlichen Grüßen}

Univ.-Prof. Dr. Dr. Max Heiland Lenkungsausschuss der DGMKG Klinik für Mund-, Kiefer- und Gesichtschirurgie, Charité Universitätsmedizin Berlin
Für Rückfragen stehe ich ger-

wollte. Bereut habe ich diese Entscheidung in keiner Phase meiner beruflichen Laufbahn.

Umso mehr erfüllt es mich mit Stolz und Dankbarkeit, mein Wissen und meine Erfahrung im Rahmen des Referats „Ästhetische Gesichtschirurgie" der DGMKG weiterzugeben.

\section{Stellenwert der MKG-Chi- rurgie in der ästhetischen Gesichtschirurgie}

$\mathrm{Ob}$ in der Traumatologie, der Tumorchirurgie, der Dysgnathiechirurgie, der Korrektur von Fehlbildungen oder auch der Implantologie - in allen diesen Kerndisziplinen der MKG-Chirurgie ist der Erfolg einer Behandlung nicht nur von der Herstellung einer optimalen Funktion, sondern auch der Wiederherstellung oder Verbesserung der Gesichtsharmonie abhängig. Die Kombination von Ästhetik und Funktion ist nicht nur eine wichtige Grundlage für die erfolgreiche Berufsausübung in der Zahnmedizin und der MKG-Chirurgie, sondern oftmals wahrscheinlich auch der Grund, weshalb viele Kollegen diesen Berufsweg eingeschlagen haben.

Um im Berufsalltag und im Wettbewerb mit anderen Fachdisziplinen erfolgreich zu sein, genügt das Erlernen der Grund- lagen allerdings nicht. Eine kontinuierliche Fortbildung und ein ständiger Blick ,über den Tellerrand" sind ebenso wichtige Voraussetzungen.

Die ästhetischen Behandlungen in der Medizin, besonders in unserem Fach, haben sich rasant weiterentwickelt, und längst sind es nicht mehr nur die chirurgischen Maßnahmen, sondern v.a. gering und non-invasive Verfahren, die den Großteil aller Behandlungen in diesem Gebiet ausmachen. Wer ausschließlich operative Verfahren anbietet, kann seine Patienten nicht umfänglich behandeln und das gewünschte Ergebnis erzielen.

\section{Aktuelle Bedeutung der Ästhetik in der MKG-Chi- rurgie}

merkmal unseres Fachs geschaffen. Das Programm ist ehrgeizig und mit sehr guten organisatorischen Strukturen ausgestattet. Das es von den Teilnehmern viel Einsatz und erhebliche finanzielle Mittel abverlangt, liegt in der Natur der Sache. Das Programm muss konsequent weiterverfolgt, wo nötig ergänzt und auch für die Öffentlichkeit sichtbarer werden.

\section{Mein persönlicher Ausblick in die Zukunft}

In meiner Tätigkeit als Leiter des Referats „Ästhetische Gesichtschirurgie" der DGMKG werde ich die Fortbildungsaktivitäten der DGMKG unterstützen und bei der

Koordination helfen. Dazu gehört,

- zusätzliche Fortbildungsmöglichkeiten in Form von Workshops und Hospitanzen zu schaffen,

Die Vielseitigkeit der MKG-Chirurgie ist für Studierende reizvoll und die umfassende Ausbildung setzt Ehrgeiz, Zielstrebigkeit und ein hohes Maß an Leistungsfähigkeit voraus. Gering und noninvasive Behandlungen der Ästhetik werden gerne anderen Fachdisziplinen überlassen. Damit schwindet in der öffentlichen Wahrnehmung die Kompetenz der MKG-Chirurgie im Bereich der Ästhetik immer mehr, v. a. natürlich bei den potenziellen Patienten. Das ist ein Teufelskreis. Mit der Einrichtung eines akkreditierten postgradualen Masterstudiengangs "Ästhetische Gesichtschirurgie“ hat die DGMKG bereits 2008 einen sehr wertvollen und unvergleichlichen Schritt in die richtige Richtung eingeschlagen und damit ein weiteres Alleinstellungs-
- Anlaufstelle für allgemeine und spezielle Fragen für den Bereich der Ästhetik zu sein,

- einen höheren Stellenwert der Ästhetik innerhalb unserer Fachzeitschrift zu sichern und - aktuelle Einschränkungen durch die Pandemie mit virtuellen Angeboten zu kontern.

In diesem Sinne freue ich mich auf eine spannende Zeit mit einem interdisziplinären und wertvollen Wissensaustausch.

Dr. Dr. Frank Muggenthaler Leiter des Referats Ästhetische Gesichtschirurgie der DGMKG Praxis für Gesichtschirurgie, Basel, Schweiz Klinik für Plastische Chirurgie und Ästhetische Medizin $\mathrm{GmbH}$, Gutach im Breisgau 


\section{Veranstaltungen}

Wiesbaden, 05.-06.02.2021
MKG-Update 2021
12. MKG-Update-Seminar
Unter der Schirmherrschaft
der DGMKG
Wiss. Leitung:
Prof. Dr. Dr. Knut A. Grötz
Prof. Dr. Andrea M. Schmidt-
Westhausen
Prof. Dr. Dr. Stefan Haßfeld
www.mkg-update.com
Infos zu allen Update-Seminaren:
med update GmbH
Tel.: 0611 - 73 65 80
Fax: 0611 - 73 65 810
info@med-update.com
Partner in der Fortbildung
me Springer Medizin
medupdate

Sehr geehrte Mitglieder, hiermit möchten wir Sie über kommende Veranstaltungen informieren.

\section{Veranstaltungen der DGMKG}

25.02.2021*

Fachkunde Strahlenschutz

Düsseldorf, 19.-21.03.2021

Curriculum Implantologie DGMKG/BDO

Düsseldorf, 19.-21.03.2021

Curriculum Parodontologie DGMKG/BDO

Mainz, 14.-16.04.2021

71. Kongress der DGMKG im Rahmen des DCK 2021

10. $-13.06 .2021^{\star}$

Praxisführungsseminar DGMKG

Berlin, 02.-04.09.2021

MKG Kompakt
Hamburg, 12.-14.11.2021

Curriculum Implantologie

DGMKG/BDO

Hamburg, 12.-14.11.2021

Curriculum Parodontologie DGMKG/BDO

München, 10.-11.12.2021

Implantologie für den Praktiker

\section{Veranstaltungen von Mitgliedern}

Aachen, 18.-20.02.2021

68th International Course for Stepwise Flap Raising

Aachen, 21.-23.02.2021

19. EUREGIO Implantationskurs am Humanpräparat

Bochum, 10.-19.03.2021

69th International Course for Flap Raising \& Microsurgery

\section{Weitere Veranstaltungen}

Maastricht/Niederlande,

31.05.-04.06.2021

TERMIS World Congress

Weitere Informationen und Anmeldeformulare finden Sie auf der DGMKG-Homepage im Veranstaltungskalender unter: https://www.dgmkg.com/veranstaltungen

*Veranstaltungsort wird noch bekannt gegeben (s. Veranstaltungskalender auf der DGMKGHomepage). 